\title{
A Comparative Study of Multiple Accessing Schemes
}

\author{
Elza Erkip Behnaam Aazhang \\ Rice University \\ Electrical and Computer Engineering-MS 366 \\ Houston, Texas 77005-1892
}

\begin{abstract}
We compare the performance of different accessing schemes (frequency division, time division and code division) for the uplink in a wireless communication system. We assume an additive white Gaussian noise channel with multipath fading. We consider the case when the receiver can track the channel parameters, but the transmitters cannot. We look at three different measures of performance: the Shannon capacity, the delay limited capacity and probability of outage. Shannon capacity is better suited for systems where the delay requirements are not as stringent, whereas the delay limited capacity and probability of outage give the performance when there are strict delay requirements or when the channel is slowly fading. We observe that all three schemes have the same achievable rates of transmission in Shannon sense, but code division multiple access scheme performs better than frequency and time division in terms of delay limited capacity and probability of outage.
\end{abstract}

\section{Introduction}

The resources available in a wireless communication system are shared between users according to several accessing schemes such as frequency division, time division or code division. The purpose of this work is to compare these accessing schemes using different measures of performance, all based on information theoretic concepts. We will focus on the uplink, which can be modeled using a multiple access channel.

The time division multiple access (TDMA) scheme allocates separate time slots for different users, while the frequency division multiple access (FDMA) scheme allocates different frequency bands. For code division multiple access (CDMA) scheme, we consider a spread spectrum system where each user is given an orthogonal code.

The comparison of achievable rate regions for TDMA and FDMA schemes in a time-invariant, memoryless additive white Gaussian noise (AWGN) chan- nel can be found in [1]. We find the rate region corresponding to orthogonal CDMA scheme by considering it as dividing the total degrees of freedom among users [2].

The mobile communication environment exhibits a randomly time varying channel with memory. The time variation and memory are due to the mobility of the users and reflection from multiple scatterers, and observed as having multiple paths and fading in the amplitude of the signal. Hence a better channel model for a wireless communication system is a multipath fading channel. We assume that the receiver can track the variations in the channel, whereas the transmitters cannot. Thus the transmitters cannot allocate power depending on the channel parameters; they have to use the same strategy independent of the channel variation.

Assuming multipath fading environment, we first compare the three accessing schemes in terms of Shannon rate regions. Other measures of performance which are better suited for slowly varying channels are the delay limited capacity [3] and the probability of outage [4]. The comparison of TDMA, FDMA and CDMA schemes according to these performance measures can be found in Section 4. We observe that in general CDMA performs better than the other schemes when time variation and delay requirements are taken into account.

\section{Time-invariant AWGN multiple ac- cess channel}

In this section we consider a continuous time multiple access channel. Without loss of generality, we restrict attention to two transmitters with equal power constraints $P$. Hence

$$
Y(t)=X_{1}(t)+X_{2}(t)+Z(t),
$$

where $X_{i}(t)$ is the signal of user $i$ and $Z(t)$ is white Gaussian noise. We assume the input signals pass through linear time-invariant ideal bandpass filters. 
The noise power spectral density is $\mathcal{N}_{0} / 2$. The frame duration is $2 T$ seconds and the signals are limited to an approximate bandwidth of $2 W$ hertz. The factor of 2 is used to be consistent with the subsequent sections. Hence the signals have $N=8 W T$ degrees of freedom per frame.

For the time division multiple access scheme, the first user transmits only in the first $\alpha 2 T$ seconds for some $0 \leq \alpha \leq 1$, while the second user transmits in the remaining $(1-\alpha) 2 T$ seconds. If the users increase their powers to $P / \alpha$ and $P /(1-\alpha)$ respectively during their transmission period, average power constraint is still satisfied. Since the users can be decoded separately, the maximum achievable rate pair $\left(R_{1}, R_{2}\right)$ in bits/second is

$$
\begin{aligned}
& R_{1}=\alpha 2 W \log \left(1+\frac{P}{\alpha 2 W \mathcal{N}_{0}}\right) \\
& R_{2}=(1-\alpha) 2 W \log \left(1+\frac{P}{(1-\alpha) 2 W \mathcal{N}_{0}}\right)
\end{aligned}
$$

By varying $\alpha$, we can get the TDMA achievable rate region.

In the FDMA scheme, both users use the channel for $2 T$ seconds but they are orthogonalized in frequency. The first user gets a frequency band of length $\alpha 2 W$, and the second uses the remaining band of length $(1-\alpha) 2 W$ for some $0 \leq \alpha \leq 1$. Using the orthogonality of the users, we can observe that the rate pair in (1) and (2) is again the maximum achievable. Hence FDMA has exactly the same capacity performance as TDMA. Although the TDMA-FDMA region is a subset of the capacity region for $\alpha=1 / 2$ optimum rate sum is achieved by both schemes. These observations were made in [1] where it was also shown that a CDMA system with non orthogonal codes and successive decoding results in the optimum capacity region.

In order to analyze the orthogonal code division multiple access scheme, we concentrate on direct sequence spread spectrum CDMA where each user is given a separate spreading code that is orthogonal to the codes of all other users. In this case both users use all the available time and bandwidth but are orthogonalized in the code dimension. We think of the orthogonal CDMA as each user getting a fraction of the $N$ total available degrees of freedom [2]. In the two user scenario, the users get $\alpha N$ and $(1-\alpha) N$ degrees of freedom respectively. Because of the orthogonality of the codes the receiver can decode the users separately, and the maximum achievable rate pair $\left(R_{1}, R_{2}\right)$ in bits/second is

$$
\begin{aligned}
& R_{1}=\frac{\alpha N}{4 T} \log \left(1+\frac{4 P T}{\alpha N \mathcal{N}_{0}}\right) \\
& R_{2}=\frac{(1-\alpha) N}{4 T} \log \left(1+\frac{4 P T}{(1-\alpha) N \mathcal{N}_{0}}\right) .
\end{aligned}
$$

This is exactly the same rate region as TDMA and FDMA when we use the fact that $N=8 W T$. Therefore all the three schemes considered have the same achievable rate region in the time-invariant case.

We next investigate the multipath fading channel model.

\section{Single user multipath fading channel}

In this section we assume multipath fading channel which is a more realistic channel model for wireless communication systems. We first consider a single user system. We assume the input signal $X(t)$ passes through a channel with random time varying impulse response $h(t, \tau)$ and is corrupted by additive white Gaussian noise $Z(t)$ with power spectral density $\mathcal{N}_{0} / 2$. The output $Y(t)$ is given by

$$
Y(t)=\int h(t, \tau) X(t-\tau) d \tau+Z(t) .
$$

We use the wide sense stationary uncorrelated scatterer model for the impulse response [5]. That is, when we view $h(t, \tau)$ as a stochastic process in $t$, it is wide sense stationary and the impulse response at different lags $\tau_{1}$ and $\tau_{2}$ are uncorrelated. The time variant frequency response $H(t, f)$ can be expressed as the Fourier transform of $h(t, \tau)$ :

$$
H(t, f)=\int h(t, \tau) e^{-2 \pi f \tau} d \tau .
$$

The spreading function $\Phi(\lambda, \tau)$ is defined as

$$
\Phi(\lambda, \tau)=\int h(t, \tau) e^{-2 \pi \lambda t} d t,
$$

and the scattering function is

$$
S(\lambda, \tau)=\mathrm{E}\left(|\Phi(\lambda, \tau)|^{2}\right) .
$$

The wide sense stationary uncorrelated scatterer assumption implies that,

$\mathrm{E}\left[\Phi\left(\lambda_{1}, \tau_{1}\right) \Phi^{*}\left(\lambda_{2}, \tau_{2}\right)\right]=S\left(\lambda_{1}, \tau_{1}\right) \delta\left(\lambda_{1}-\lambda_{2}\right) \delta\left(\tau_{1}-\tau_{2}\right)$.

The multipath spread $T_{m}$ of the channel is the largest $\tau$ such that $S(\lambda, \tau)$ is nonzero. Similarly, the largest spread of $S(\lambda, \tau)$ is called the Doppler spread and denoted by $B_{d}$. For a time-invariant channel the 
Doppler spread is zero. See [5] for a thorough treatment of these concepts.

We assume the signals that are transmitted over this channel are approximately bandlimited to $W$ hertz, and frame duration is $T$ seconds. The average power constraint of the transmitter is $P$ watts. The channel is slowly fading frequency non-selective if $W T_{m} \ll 1$ and $T B_{d} \ll 1$. This means that the time variant frequency response $H(t, f)$ is essentially constant over the bandwidth $W$ and over the time period $T$ and can be approximated by $H=H(0,0)$. Then for a particular realization of the channel frequency response the maximum mutual information over one frame becomes

$$
C=W \log \left(1+\frac{P|H|^{2}}{W \mathcal{N}_{0}}\right) \text { bits/second. }
$$

The Shannon capacity $C_{S}$ is found by averaging $C$ over a large number of frames. Assuming a stationary and ergodic channel, this average is equal to the expected value of $C[6]$. Thus

$$
C_{S}=\mathrm{E}_{H}(C)=\mathrm{E}_{H}\left\{W \log \left(1+\frac{P|H|^{2}}{W \mathcal{N}_{0}}\right)\right\} .
$$

In order to achieve Shannon capacity we need a large number of frames so that we can observe the ergodicity of the channel. In particular, when the variations in the channel are very slow, the coding delay to achieve Shannon capacity can be very long, as this delay is needed to average out the channel parameters as well as the noise in the system. The delay limited capacity [3] is defined by imposing restrictions on the coding delay for different fading processes and like Shannon capacity it only depends on the stationary distribution of $h(t, \tau)$. The delay limited capacity is the maximum transmission rate such that the same coding delay provides reliable transmission for all stationary, ergodic fading processes having the same stationary distribution. The delay limited capacity is more relevant for systems where large delays cannot be tolerated, and the fading is very slow. Following [3], the delay limited capacity $C_{D}$ can be written as

$$
C_{D}=\min _{H \in \mathcal{H}} C=\min _{H \in \mathcal{H}}\left\{W \log \left(1+\frac{P|H|^{2}}{W \mathcal{N}_{0}}\right)\right\}
$$

where $\mathcal{H}$ is the set of all possible frequency responses $H(t, f)$. Whenever the frequency response $H=0$ is in the support of the fading process (which is the case for almost all of the practical fading models used), the delay limited capacity is zero. Note that as the transmitter cannot track the channel variations, the power cannot be allocated into different fading states. In [3], where it was assumed that the transmitter can observe the channel state and allocate power optimally, it was possible to get nonzero delay limited capacity for a wide class of channels. Hence we argue that the delay limited capacity may not be best suited for the problem considered in this study and gives us a very pessimistic measure of performance.

The third measure of performance that is suitable for slowly fading channels with severe delay limitations is the probability of outage [4]. If we can only use a small number of frames, we cannot talk about expected values and the mutual information fluctuates. In that case we declare outage if the average mutual information over the required number of frames falls below a certain rate $\alpha$. We can write the probability of outage as

$$
P_{\text {out }}=\operatorname{Prob}\left(\frac{1}{M} \sum_{i=1}^{M} C_{i}<\alpha\right),
$$

where $M$ is the number of frames allowed and $C_{i}$ is the maximum mutual information over frame $i$ given by

$$
C_{i}=W \log \left(1+\frac{P\left|H_{i}\right|^{2}}{W \mathcal{N}_{0}}\right)
$$

with $H_{i}=H(i T, 0)$. Although $T B_{d} \ll 1$, we are not guaranteed to have $M T B_{d}<1$, and therefore the frequency response $H(t, f)$ might vary from one frame to another and result in possibly different $H_{i}$.

When $2 M W T \gg 1$, if for a certain realization of the channel we have $1 / M \sum_{i=1}^{M} C_{i} \geq \alpha$, then we will be able to send at an average rate of $\alpha$ bits/second reliably over $M$ frames. However in the event of an outage reliable communication is no longer possible.

We next concentrate on the uplink and investigate TDMA, FDMA and CDMA schemes in terms of our three different measures of performance.

\section{Multipath fading multiple access channel}

We now consider a multiuser system in a multipath fading environment. We have a $K$ user multiple access channel. Each user has equal average power $P$, and user $k$ 's transmitted signal $X_{k}(t)$ goes through a multipath fading channel $h^{(k)}(t, \tau)$ for $k=1, \ldots, K$. Furthermore, these signals are corrupted by additive white Gaussian noise $Z(t)$ of power spectral density $\mathcal{N}_{0} / 2$. Therefore the output of the channel $Y(t)$ is

$$
Y(t)=\sum_{k=1}^{K} \int h^{(k)}(t, \tau) X_{k}(t-\tau) d \tau+Z(t)
$$


We assume all the channels have roughly the same multipath spread $T_{m}$ and Doppler spread $B_{d}$.

We consider a frame duration of $K T$ seconds, and a bandwidth of $K W$ hertz. We assume that $W T_{m} \ll 1$ and $T B_{d} \ll 1$, but not necessarily $K W T_{m}<1$ or $K T B_{d}<1$. If we consider the time frame $K T$ to be divided into $K$ slots of duration $T$ and the total bandwidth $K W$ into $K$ frequency bands of length $W$, then the channel frequency responses $H^{(k)}(t, f)$ are roughly constant over each slot and band, but there might be variations within the total duration of a frame or within the total bandwidth. We will denote the frequency response of channel $k$ over time slot $i$ and frequency band $j$ as $H^{(k)}(i, j)$. These assumptions are similar to those made in [6].

We first consider a TDMA scheme where each user is assigned a time slot of length $T$ within a frame. Hence the frequency response of channel $k$ over the time slot of user $k$ (call this slot $k$ ) is roughly timeinvariant. However, we need to average over the $K$ different frequency bands as each transmitter uses the whole frequency band and the channel is frequency selective over the total bandwidth $K W$. User $i$ divides $P K T$, the the total energy of the transmitted signal, over these $K$ frequency bands equally. As the transmitters cannot track the channel variations, this is the best strategy. Then for a particular realization of the frequency response the maximum mutual information for user $k$ over frequency band $j$ is

$$
\frac{W}{K} \log \left(1+\frac{P\left|H^{(k)}(k, j)\right|^{2}}{W \mathcal{N}_{0}}\right) .
$$

Note that the multiplicative factor is $W / K$ as the frame period is $K T$ seconds. Summing this over the $K$ frequency bands we get the maximum achievable rate for user $k$ as

$$
C^{\mathrm{TDMA}, k}=\frac{1}{K} \sum_{j=1}^{K} W \log \left(1+\frac{P\left|H^{(k)}(k, j)\right|^{2}}{W \mathcal{N}_{0}}\right) .
$$

The FDMA scheme is similar; now all the users use the channel during the whole frame, but they are orthogonalized in frequency. User $k$ gets frequency band $k$. Since each frequency band has width of $W$ hertz, the channel impulse response $H^{(k)}(t, f)$ is roughly constant in frequency, but might vary in time. In this case user $i$ divides the total energy $P K T$ over the $K$ time slots equally, and for a particular realization of the frequency response the maximum mutual information for user $k$ over time slot $j$ is

$$
\frac{W}{K} \log \left(1+\frac{P\left|H^{(k)}(j, k)\right|^{2}}{W \mathcal{N}_{0}}\right) .
$$

We sum this term over time slots $j$ to get the maximum achievable rate for user $k$ as

$$
C^{\mathrm{FDMA}, k}=\frac{1}{K} \sum_{j=1}^{K} W \log \left(1+\frac{P\left|H^{(k)}(j, k)\right|^{2}}{W \mathcal{N}_{0}}\right) .
$$

For the CDMA scheme, we have orthogonal spreading codes for different users. As in the time-invariant case, we think of this as giving each user separate degrees of freedom. Recall that we have $K$ time slots of duration $T, K$ frequency bands of length $W$ and the frequency responses of the channels can be considered roughly constant over each frame and slot. There are $K^{2}$ combinations of the time slots-frequency bands and each combination provides $2 W T$ degrees of freedom. We divide these $2 W T$ degrees of freedom equally among the $K$ users. Therefore each user gets $2 W T / K$ degrees of freedom per slot per band. Every user divides the total energy $P K T$ into these $K^{2}$ time slotfrequency band pairs equally, thus allocating $P T / K$ to each. Therefore, the maximum mutual information for user $k$ in time slot $i$ frequency band $j$ is

$$
\frac{W}{K^{2}} \log \left(1+\frac{P\left|H^{(k)}(i, j)\right|^{2}}{W \mathcal{N}_{0}}\right) .
$$

The maximum achievable rate for user $k$ for a particular realization of the channel is obtained as the sum over all the $K^{2}$ time slot-frequency band pairs:

$$
C^{\mathrm{CDMA}, k}=\frac{1}{K^{2}} \sum_{i=1}^{K} \sum_{j=1}^{K} W \log \left(1+\frac{P\left|H^{(k)}(i, j)\right|^{2}}{W \mathcal{N}_{0}}\right) .
$$

We are now in a position to compare the three accessing schemes according to the three different measures of performance. We first consider the Shannon capacity. The wide sense stationary and uncorrelated scatterer assumption imply that the distribution of $H^{(k)}(t, f)$ is independent of $t$ and $f$ for the Gaussian model [5]. Therefore $H^{(k)}(i, j)$ has the same distribution for all pairs $(i, j)$. Let $H^{(k)}$ be a random variable which has this common distribution. However, recall that the channel is frequency selective; for any realization $H^{(k)}(i, j)$ might take on different values for different $(i, j)$.

The Shannon capacity is given by the expectation of maximum mutual information over all realizations of the channel frequency response. Taking the expectation of (6), (7) and (8) we find the Shannon capacities of the accessing schemes for user $k$ as

$$
\begin{aligned}
C_{S}^{\mathrm{TDMA}, k} & =C_{S}^{\mathrm{FDMA}, k}=C_{S}^{\mathrm{CDMA}, k} \\
& =\mathrm{E}_{H}\left\{W \log \left(1+\frac{P\left|H^{(k)}\right|^{2}}{W \mathcal{N}_{0}}\right)\right\} .
\end{aligned}
$$


As in the time-invariant case all the accessing schemes have the same performance in Shannon capacity sense. Shannon capacity measures the long term behavior, and in the long range all the time slots and frequency bands have similar characteristics which result in the same Shannon capacity for TDMA, FDMA and CDMA. However, when long coding delays cannot be tolerated or when the time variation of the system is slow, the Shannon capacity does not give us a realistic measure of performance.

We next concentrate on the delay limited capacity. In this case the coding delay is the number of frames used and the delay limited capacity for user $k$ is given as the minimum of $C^{k}$ (corresponding to the particular accessing scheme) over all possible realizations of the frequency response $H^{(k)}(t, f)$. Note that $H^{(k)}(t, f)$ is defined on the whole frame duration $K T$ and the total bandwidth $K W$. Since the distribution of $H^{(k)}(t, f)$ is independent of $t$ and $f$,

$$
\min _{H^{(k)}(i, j)}\left\{W \log \left(1+\frac{P\left|H^{(k)}(i, j)\right|^{2}}{W \mathcal{N}_{0}}\right)\right\}
$$

will be independent of the pair $(i, j)$. However, because of the correlation between time slots and between frequency bands,

$$
\begin{gathered}
\min _{H^{(k)}}\left\{\frac{1}{K^{2}} \sum_{i=1}^{K} \sum_{j=1}^{K} W \log \left(1+\frac{P\left|H^{(k)}(i, j)\right|^{2}}{W \mathcal{N}_{0}}\right)\right\} \geq \\
\min _{H^{(k)}}\left\{\frac{1}{K} \sum_{j=1}^{K} W \log \left(1+\frac{P\left|H^{(k)}(k, j)\right|^{2}}{W \mathcal{N}_{0}}\right)\right\} \geq \\
\min _{H^{(k)}}\left\{W \log \left(1+\frac{P\left|H^{(k)}(i, j)\right|^{2}}{W \mathcal{N}_{0}}\right)\right\} .
\end{gathered}
$$

Therefore,

$$
C_{D}^{\mathrm{CDMA}, k} \geq C_{D}^{\mathrm{TDMA}, k}
$$

and similarly

$$
C_{D}^{\mathrm{CDMA}, k} \geq C_{D}^{\mathrm{FDMA}, k}
$$

Hence CDMA performs better than TDMA or FDMA in delay limited sense. The comparison of TDMA and FDMA depends on the particular channel model. Unfortunately, as noted in the single user analysis, the delay limited capacity is equal to zero for most of the practical channel models used. We feel the delay limited capacity with power control at the transmitters will provide better insight into the comparison.

Finally we investigate the probability of outage for the different accessing schemes. The analytical calculation of $P_{\text {out }}$ is difficult for most channel models

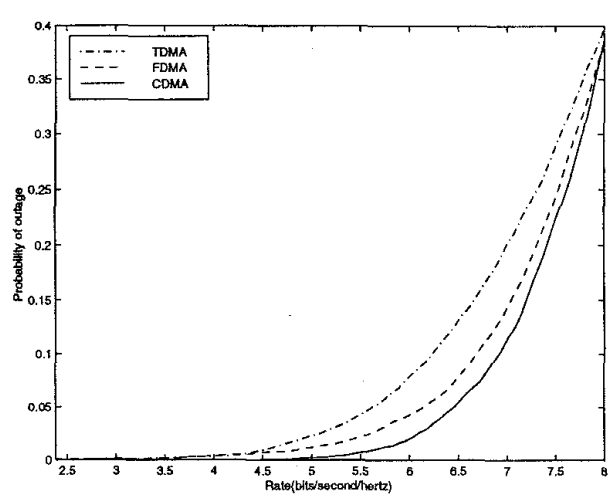

Figure 1: Probability of outage versus information rate for $\mathrm{SNR}=20 \mathrm{~dB}$

and we provide simulation results. Within the frame period of $K T$ seconds and bandwidth of $K W$ hertz, the $k$ 'th channel impulse response $h^{(k)}(t, \tau)$ can be expressed as $[5,7]$

$\frac{1}{K^{2} W T} \sum_{l=0}^{L-1} \sum_{r=0}^{R-1} \Phi^{(k)}\left(\frac{r}{K T}, \frac{l}{K W}\right) \delta\left(\tau-\frac{l}{K W}\right) e^{\frac{2 \pi r t}{K T}}$

where $L=\left\lceil K W T_{m}\right\rceil, R=\left\lceil K T B_{d}\right\rceil$, and $\Phi^{(k)}(\lambda, \tau)$ is the spreading function of channel $k$ defined in (3). For the Rayleigh fading multipath channel, the coefficients $\Phi^{(k)}(r / K T, l / K W)$ are complex Gaussian random variables, and by (5) they are independent. The scattering function $S(\lambda, \tau)$ in (4) provides us the variances of these coefficients. For the $\lambda$ variation we use Jakes' model [8] and for the $\tau$ variation we use one sided exponential profile [9]. Hence

$$
S(\lambda, \tau) \propto \frac{1}{\sqrt{B_{d}^{2}-\left(2 \lambda-B_{d}\right)^{2}}} e^{-7 \tau / T_{m}}
$$

for $0<\lambda<B_{d}, 0<\tau<T_{m}$ and zero otherwise. Note that we have shifted the original Jakes' model by $B_{d} / 2$ in order to have the $\lambda$ support of $S(\lambda, \tau)$ be limited to $\left(0, B_{d}\right)$.

Using the above channel model, we calculate the probability of outage of user $k$ over one frame as

$$
P_{\text {out }}=\operatorname{Prob}\left(C^{k}<\alpha\right)
$$

for all the accessing schemes using a signal to noise ratio $\left(P / W \mathcal{N}_{0}\right)$ of $20 \mathrm{~dB}$. The probability of outage versus information rate $\alpha$ is plotted in Figure 1 . We are generally interested in low probability of outage. 


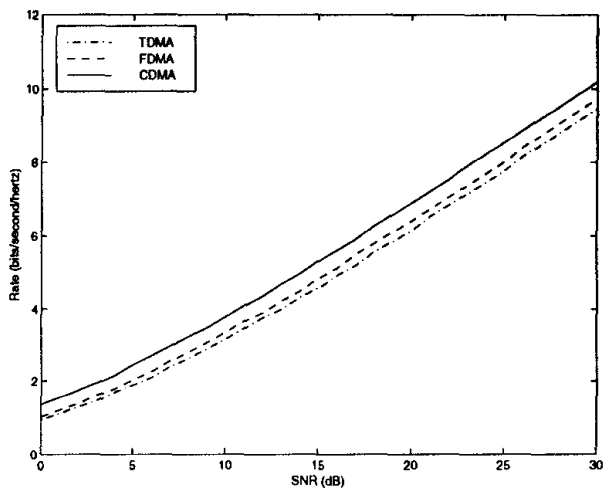

Figure 2: Information rate versus SNR for $P_{\text {out }}=0.05$

We observe that CDMA has better performance than TDMA and FDMA in terms of probability of outage for the same signal to noise ratio (SNR).

We also plot information rate $\alpha$ versus SNR for $P_{\text {out }}=0.05$ in Figure 2. This plot shows that the SNR required for CDMA to achieve rate $\alpha$ with probability of outage 0.05 is smaller than the SNR required for TDMA or FDMA. In fact for large SNR the capacity versus SNR curves are roughly parallel. The relative performance of TDMA and FDMA depend on the scattering function used, $L$ and $R$. In fact for identity scattering function, their performance is identical for same number of multipath (L) and Doppler (R) components, but still worse than that of CDMA.

We would like to note that although we investigated the uplink, current implementation of downlink systems can be analyzed similarly. Hence we conclude that CDMA performs better than TDMA and FDMA in delay limited sense and in probability of outage for current downlink implementations.

\section{Conclusions}

In this study we have compared different multiple accessing schemes over a multipath fading multiple access channel according to three measures of performance. The first measure of performance was the Shannon capacity which gives long term achievable communication rates, but does not capture the time scale of variations in the channel. We have observed that time division, frequency division and code division access schemes have the same Shannon capacities. The second measure of performance, the delay limited capacity, is mostly used for slowly varying systems with strict delay requirements. We observed that CDMA performs better than TDMA and FDMA in the delay limited sense. However, without any power control at the transmitters, the delay limited capacity may be very pessimistic. The third measure was given by the probability of outage, which is also appropriate for delay sensitive applications. We observed that CDMA has better probability of outage performance than TDMA and FDMA. We argue that code division systems might be better suited for mobile environments where the fading is slow and long coding delays cannot be tolerated.

\section{References}

[1] T. M. Cover and J. A. Thomas, Elements of Information Theory, John Wiley \& Sons, 1991.

[2] J.L. Massey, "Towards and Information Theory of Spread-Spectrum Systems," in S.G. Glisic and P.A. Leppanen, editors, Code Division Multiple Access Communications, pp. 29-46, Kluwer Academic Publishers, 1995.

[3] S. V. Hanly and D. N. Tse, "Multi-Access Fading Channels: Part II: Delay limited capacities", submitted to IEEE Transactions on Information Theory.

[4] L. H. Ozarow, S. Shamai and A. D. Wyner, "Information Theoretic Considerations for Cellular Mobile Radio," IEEE Transactions on Vehicular Technology, Vol, 43, pp. 359-378, 1994.

[5] J. G. Proakis, Digital Communications, McGrawHill, 1995.

[6] R. Gallager, "An Inequality on the Capacity Region of Multiaccess Fading Channels," in Communications and Cryptography-Two Sides of One Tapestry, pp. 129-139, Kluwer, 1994.

[7] A. M. Sayeed and B. Aazhang, "Joint MultipathDoppler Diversity in Mobile Wireless Communications," submitted to IEEE Transactions on Communications.

[8] W. C. Jakes, "Multipath Interference," in W. C. Jakes, editor, Microwave Mobile Communications, pp. 11-78, IEEE Press, 1993.

[9] R. L. Peterson, R. E. Ziemer and D. E. Borth, Introduction to Spread Spectrum Communications, Prentice-Hall, 1995. 